\section{BRAZIULIAN JOURNAL \\ OF MEDICAL AND BIOLOCICAL RESFARCH}

www.bjournal.com.br
ISSN 0100-879X

Volume 43 (8) 698-811 August 2010

BIOMEDICAL SCIENCES

AND

CLINICAL INVESTIGATION

Braz J Med Biol Res, August 2010, Volume 43(8) 745-758

doi: 10.1590/S0100-879X2010007500070

Lateral asymmetry of voluntary attention orienting

B.A. Castro-Barros, A.M. Lacerda, L.L. Righi and L.E. Ribeiro-do-Valle

The Brazilian Journal of Medical and Biological Research is partially financed by
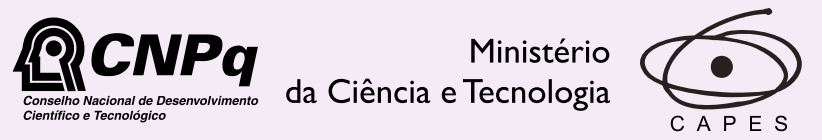

Ministério da Educação

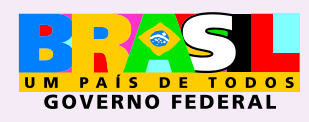

DTAPESP

Institutional Sponsors
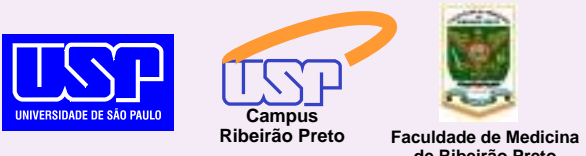

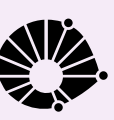

UNICAMP $\oplus$ SHIMADZU

GE Healthcare
Hotsite of proteomics metabolomics developped by: 


\title{
Lateral asymmetry of voluntary attention orienting
}

\author{
B.A. Castro-Barros, A.M. Lacerda, L.L. Righi and L.E. Ribeiro-do-Valle \\ Departamento de Fisiologia e Biofísica, Instituto de Ciências Biomédicas, \\ Universidade de São Paulo, São Paulo, SP, Brasil
}

\begin{abstract}
We recently demonstrated that automatic attention favors the right side of space and, in the present study, we investigated whether voluntary attention also favors this side. Six reaction time experiments were conducted. In each experiment, 12 new 18-25-year-old male right-handed individuals were tested. In Experiments 1, 2, 3 (a, b) and 4 (a, b), tasks with increasing attentional demands were used. In Experiments 1, 2, 3a, and 4a, attention was oriented to one or both sides by means of a central spatially informative visual cue. A left or right side visual target appeared 100, 300, or 500 ms later. Attentional effects were observed in the four experiments. In Experiments 2, 3a and 4a, these effects were greater when the cue indicated the right side than when it indicated the left side (respectively: $16 \pm 10$ and $44 \pm 6 \mathrm{~ms}, P=0.015$, for stimulus onset asynchrony of $500 \mathrm{~ms}$ in Experiment 2; $38 \pm 10$ and $70 \pm 7 \mathrm{~ms}, \mathrm{P}=0.011$, for Experiment 3a, and $23 \pm 11$ and $61 \pm 10 \mathrm{~ms}, \mathrm{P}=0.009$, for Experiment 4a). In Experiments $3 \mathrm{~b}$ and $4 \mathrm{~b}$, the central cue pointed to both sides and was said to be non-relevant for task performance. In these experiments right and left reaction times did not differ. The most conservative interpretation of the present findings is that voluntary attention orienting favors the right side of space, particularly when a difficult task has to be performed.
\end{abstract}

Key words: Voluntary attention; Visuospatial attention; Lateral asymmetry; Reaction time tasks

\section{Introduction}

Attention may be considered to be the neural activity, which allows people to "selectively process information in the environment that is relevant to their behavioral goals" (1). The processing of a selected stimulus or stimuli in a selected spatial region is facilitated and/or the processing of concurrent stimuli is inhibited (2-15).

Two kinds of attention have been distinguished in the literature, stimulus-driven or automatic (also called exogenous or bottom-up) and goal-directed or voluntary (also called endogenous or top-down) (see Ref. 16). Automatic attention is captured by unexpected, intense or significant peripheral stimuli. Voluntary attention is oriented by symbolic cues, in accordance with current personal interests or requests of others.

Evidence exists that automatic visual attention favors the right side of space (17-20). This right side favoring has been related to the hemispheric asymmetry of the mechanisms responsible for this kind of attention. While the left hemisphere mediates automatic attention to the right side of space, the right hemisphere mediates automatic attention to the left and right sides of space (21-24). The double control of automatic attention to the right side, as opposed to the single control of automatic attention to the left side, might increase its action on the right side.

The mechanisms responsible for voluntary visual attention are also asymmetrically distributed between the two hemispheres, with the left hemisphere controlling orientation to the right side and the right hemisphere controlling orientation to both sides (21-23,25; see also Ref. 2); the magnitude of this asymmetry, however, seems to be smaller than that for automatic attention.

The larger neural network (networks of both the left and right hemispheres), which controls voluntary attention to the right side of space could be expected to provide more attentional resources than the smaller neural network (network of only the right hemisphere), which controls voluntary attention only to the left side of space. This expected difference in the amount of resources from the two networks could lead to a favoring of the right side by voluntary attention, particularly in the case of more difficult tasks. In these tasks, more attentional resources are required to process the stimuli and the neural network

Correspondence: L.E. Ribeiro-do-Valle, Departamento de Fisiologia e Biofísica, ICB, USP, 05508-000 São Paulo, SP, Brasil.

E-mail: ribeiro@icb.usp.br

Received December 31, 2009. Accepted July 6, 2010. Available online July 23, 2010. Published August $13,2010$. 
controlling attention to the right side should be more able to provide these extra resources than the neural network controlling attention to the left side. Considering the smaller hemispheric asymmetry of voluntary attention mechanisms, this favoring should be less conspicuous than that evidenced for automatic attention.

Most studies in which the action of voluntary attention on behavior was investigated found no lateral asymmetry (e.g., 21,23). In the study by Corbetta et al. (21), attention was oriented sequentially along a leftward or a rightward direction, to each one of four peripheral locations in the left or right visual field. A simple visual stimulus to which the volunteers should respond with their left hand appeared most of the times in the attended location and a few times in any one of the other locations. Although reaction times to stimuli presented in validly cued locations were faster than reaction times to stimuli presented in the other locations, no consistent effect of visual field was observed. In the study by Nobre et al. (23), attention was oriented to a peripheral location contralateral to that where a prime stimulus occurred. Only a non-significant reaction time difference between the two sides was reported. Some evidence for a lateral asymmetry was obtained by Barthelemy and Boulinguez (26), Rhodes and Robertson (27) and Evert et al. (28). The first two groups $(26,27)$ obtained results compatible with a left side favoring by voluntary attention and the latter group (28) obtained results compatible with a right side favoring by voluntary attention.

Barthelemy and Boulinguez (26) tested their volunteers using a location identification reaction time task. Responses were given with the thumb or index finger of the same hand (the left hand was used in half of the blocks of trials and the right hand in the other half) on buttons arranged on each side of the sagittal axis of the body. Attention was directed to the left or right visual hemifield by means of a central cue. As expected, reaction times were shorter in the valid condition than in the neutral condition, and shorter in this condition than in the invalid condition. A left hand advantage in the neutral and invalid conditions was observed. More important, the predicted stimulus-response spatial compatibility effect occurred for both the left and the right hand in the valid condition but only for the left hand in the neutral condition. The authors attributed the absence of the compatibility effect for the right hand in this last condition to an arousal dominance by the right hemisphere, which would compensate for the influence of compatibility. An alternative explanation could be a left side favoring by voluntary attention, expressed because of a competition between the leftward and the rightward orienting mechanisms in the neutral condition.

Evert et al. (28) also used a letter identification reaction time task and a valid, invalid or neutral central cue. In their first experiment, the target stimulus (an "M" or a "W") was presented alone and in their second experiment, simultaneously with a distractor stimulus (an "X"). Similar between-hemifield benefits and no costs were observed in the former experiment. In the second experiment, the valid cue also produced a benefit, which was similar for the two visual hemifields; the invalid cue produced no cost when pointing to the right visual hemifield but produced a large cost when pointing to the left visual hemifield. This latter finding might be interpreted to indicate a stronger inhibition of right side stimulus processing, which would correspond to a left side favoring by voluntary attention.

Rhodes and Robertson (27) required their volunteers to differentiate a normally oriented letter from a mirrorreflected letter. The target stimulus was preceded by a valid, an invalid or a neutral central cue. They observed a shorter reaction time to the target on the right side than on the left side for the valid and neutral conditions, but no difference between reaction times to the target on the two sides for the invalid condition. At first glance, these results are compatible with the idea of a favoring of the right side by voluntary attention. However, if the linguistic nature of the target stimuli used by the authors favored their processing on the right side (see Ref. 29), the opposite conclusion would be more appropriate.

At present, it is apparent that no clear-cut evidence exists supporting the idea that voluntary attention tends to favor the right side of space. If anything, the opposite would seem more likely, whatever the reason.

In a recent study, Castro-Barros et al. (17) presented solid evidence for the existence of a right-side favoring by automatic visual attention. They used two kinds of tasks. In one of them, the volunteers had to detect a peripheral simple stimulus (a small vertical line) and respond to it with the hand on the same side. In the other, they had to discriminate the same target from a no-go target and also respond with the hand on the same side. In both tasks a prime stimulus preceded the target by 34 to $100 \mathrm{~ms}$. The target could occur on the same location as the prime stimulus or on a symmetric contralateral location. In three of the four experiments, greater effects were produced by the right side prime stimulus than by the left side prime stimulus when stimulus onset asynchrony was $100 \mathrm{~ms}$. The authors concluded that the appearance of this lateral asymmetry was due to the fact that the target stimulus was difficult to detect and identify. More attention would have been required to perform the task and the right hemisphere rightward orienting attentional mechanism would possibly have been mobilized to the point of influencing behavior. In addition, the two tasks that were used probed sensorimotor processing related to each side of space in turn. This should also have facilitated the expression of any existing lateral asymmetry of automatic attention action. The nonlinguistic nature of the target stimulus used allows one to exclude the hemispheric asymmetry of language mechanisms as being responsible for the finding.

Following the line of reasoning of Castro-Barros et al. (17), one could attribute the failure so far to observe a 
right-side of space favoring by voluntary attention to the low attentional demand of the tasks, which have been commonly used. In these tasks, the right hemisphere rightward-orienting attentional mechanism would not have been mobilized to a significant extent. Perhaps by using more difficult tasks, which demand more attention and could involve a stronger mobilization of this mechanism to provide it, such a favoring would be demonstrable.

The present study tested the hypothesis that voluntary attention tends to favor the right side of space.

The Ethics Committee of the Biomedical Sciences Institute of São Paulo University approved this study. Written informed consent was obtained from all participants.

\section{Experiment 1}

This experiment investigated whether the hypothesized right-side favoring by voluntary attention would appear in an experimental situation demanding a relatively low level of attention, requiring, presumably, little involvement of the right hemisphere rightward orienting attentional mechanism. The volunteers had to identify the side (left or right) of appearance of the target stimulus and respond to it with the hand on the same side. In two-thirds of the trials, a central cue correctly indicated the side of the impending target. In one-sixth of the trials, the central cue incorrectly indicated the side of the impending target. In the remaining sixth of the trials, a bilaterally pointing cue indicated that the target could occur equally on any side. This last condition was intended to induce some competition between the two sides for voluntary attention orienting.

A favoring of the right side by voluntary attention should be revealed by a shorter reaction time to the target on the right side than on the left side for the valid trials and/or a longer reaction time to the target on the left side than on the right side for the invalid trials. It might also be manifested by a shorter reaction time to the target on the right side than on the left side for the bilateral cue trials given that, in this condition, the mechanisms that orient attention to the right side might prevail. An earlier appearance of the effects of right side-oriented attention might occur.

\section{Methods}

Participants. Twelve naive young adult males voluntarily participated in the experiment. They were right-handed according to the Edinburgh Inventory (30) and presented normal or corrected-to-normal vision. None of them possessed previous experience with reaction time tasks or were aware of the purpose of the study.

Apparatus. The participants were tested in a dimly illuminated and sound-attenuated room. Their head was positioned in a chin-and-front rest so that their eyes remained $57 \mathrm{~cm}$ away from the screen of a 15-inch video monitor, set to work with a refresh rate of $60 \mathrm{~Hz}$. The background color of this screen was white and its luminance was 26.9 $\mathrm{cd} / \mathrm{m}^{2}$. There was a central black fixation point $\left(0.1 \mathrm{~cd} / \mathrm{m}^{2}\right)$ and two gray rings $\left(8.7 \mathrm{~cd} / \mathrm{m}^{2}, 1.50\right.$ degrees in diameter and with a 0.04-degree wide margin) centered 9 degrees away to the left and right of this fixation point (Figure 1). The participants were instructed to keep their eyes on the fixation point and to respond to stimuli presented on the marked locations. The arms of the participants rested on a table and their left and right index fingers, respectively, touched a left side and a right side optic switch key mounted on top of this table. An IBM-compatible computer controlled by a program developed with the MEL2 software (Psychology Software Tools Inc., USA) generated the stimuli and recorded the responses.

Procedure. Each participant was tested in two sessions on separate days, no more than seven days apart. Before each session, they read a brief explanation about the task. A more detailed explanation was given in the testing room, where they were able to watch the stimuli on the screen and press the response keys. The participant was then asked to perform about 20 practice trials to verify his comprehension of the task.

The first testing session consisted of one block with 72 trials. Its finality was to familiarize the volunteers with the task. Each trial began with the appearance of the fixation point and the two gray rings. Between 1850 and $2350 \mathrm{~ms}$ later, a central cue was presented. This cue had the shape of an arrowhead, formed by two converging lines 0.45 degrees in length and a 0.04-degree wide margin, was gray in color and had luminance of less than $0.1 \mathrm{~cd} / \mathrm{m}^{2}$. This arrowhead pointed to the left hemifield in $41.67 \%$ of the trials and to the right hemifield in $41.67 \%$ of the trials. In the remaining

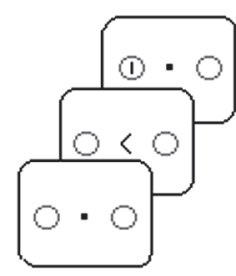

Valid cue

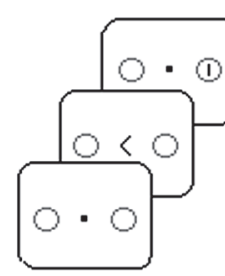

Invalid cue

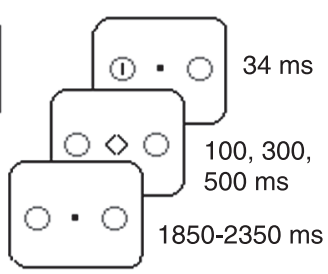

Biateral cue
Figure 1. Diagrammatic representation of the sequence of visual displays in each cueing condition (valid, invalid and bilateral). The trial starts with the appearance of the central fixation point and the two peripheral rings, which indicate the places where the target stimuli can occur. After 1850 to 2350 ms, the cue, represented by a left, right or left and right pointing arrows, replaces the fixation point. This cue remains on the screen for 100,300 or $500 \mathrm{~ms}$. The target stimulus, represented by a vertical line in Experiments 1 and $3 a$ and $3 b$ and by this vertical line or a small ring in Experiments 2 and $4 \mathrm{a}$ and $4 \mathrm{~b}$, appears immediately after the disappearance of the cue, and lasts for $34 \mathrm{~ms}$. The single arrow cue occurs in $84 \%$ of the trials; in $80 \%$ of these trials the target stimulus occurs at the side indicated by it and in the remaining trials, at the opposite side. The double arrow cue occurs in $16 \%$ of the trials; the target stimulus occurs in the left side in $50 \%$ of these trials and in the right side in the remaining trials. 
$16.67 \%$ of the trials there were two arrowheads, one pointing to the left hemifield and the other to the right hemifield. Each of these cues could last 100, 300, or 500 ms, being equally probable and randomly distributed. Immediately after the disappearance of the cue, a 34-ms target stimulus, represented by a black vertical line ( 0.45 degrees in length and a 0.04-degree wide margin), with a luminance of less than $0.1 \mathrm{~cd} / \mathrm{m}^{2}$, appeared inside one of the peripheral rings. In the trials in which one arrowhead was presented, this target occurred in the hemifield indicated by the arrow with a probability of $80 \%$ (valid cue trials) and in the opposite hemifield, with a probability of $20 \%$ (invalid cue trials). In the remaining trials, in which the two arrowheads were presented, the target occurred half of the time in the left hemifield and half of the time in the right hemifield (bilateral cue trials). The participant was instructed to pay attention to the side or sides indicated by the cue and to respond as fast as possible to the target with the same side hand. As soon as the response was given or an interval of 600 ms had elapsed, a message appeared for $400 \mathrm{~ms}$ over the fixation point. The reaction time, in milliseconds, appeared when the participant responded between 150 and $600 \mathrm{~ms}$ after the onset of the target. The message "anticipated" or "slow" was displayed when the participant emitted a response less than $150 \mathrm{~ms}$ after the onset of the target or more than $600 \mathrm{~ms}$ after the onset of the target, respectively. The message "incorrect" was displayed when the participant responded between 150 and $600 \mathrm{~ms}$ with the opposite side hand. Then, the next trial began. The purpose of this first testing session was to familiarize the volunteers with the entire experimental situation.

The second session was similar to the first. It consisted of six blocks, each with 72 trials. A rest interval was provided between one block and the next, the duration of which was controlled by the participant. A green asterisk replaced the reaction time message and a red asterisk replaced the error messages.

In this second session, each of the "valid cue" conditions occurred a total of 48 times, each of the "invalid cue" conditions occurred a total of 12 times, and each of the "bilateral cue" conditions occurred a total of 12 times. The relatively small number of trials in these last two conditions was a deliberate choice made to avoid the reaction time variability caused by the fatigue and lack of motivation that normally occur in long testing sessions. It should have been compensated for by the precise recording of the responses (the joystick port of the computer was used for signal input) and the familiarization with the task that occurred in the first testing session, which reduce reaction time variability.

Data analysis. Reaction time was the dependent variable. The mean of the six reaction time block medians in the second session, for each condition, was calculated for each participant. The number of anticipated responses, slow responses and incorrect responses in the same session, for each condition, was also evaluated for each participant.
Reaction time data were submitted to repeated measures analysis of variance (ANOVA), with the stimulus onset asynchrony (SOA) $(100,300$, or $500 \mathrm{~ms})$, type of cue (valid, invalid or bilateral) and hemifield of appearance of the target (left or right) as factors. When appropriate, these data were further analyzed by the Newman-Keuls test. Of great interest was the comparison between reaction time to the target in the left hemifield after the cue indicated this hemifield and reaction time to the target in the right hemifield after the cue indicated this hemifield, which might reveal a lateral asymmetry of the facilitatory action of attention. Of equal interest was the comparison between reaction time to the target in the right hemifield after the cue indicated the left hemifield and reaction time to the target in the left hemifield after the cue indicated the right hemifield, which might reveal a lateral asymmetry of the inhibitory action of attention. Also of interest was the comparison between reaction time to the target in the right hemifield and reaction time to the target in the left hemifield after the cue indicated both hemifields; in this case, the lateral competition for attention action might reveal a favoring of one side.

It should be noted that, because one side might be favored by attention when the cue indicated both hemifields, there was no true neutral condition in the testing sessions. Because of that no cost-benefit analysis was performed on the data.

The level of significance was set at 0.05 for all analyses.

\section{Results}

The percentages of anticipation, inversion and omission errors were $2.9,0.7$, and 0.9 , respectively.

A main effect of SOA [F2,22 $=93.12, \mathrm{P}<0.001]$ and of type of cue $[F 2,22=57.82, P<0.001]$ occurred. No main effect of target hemifield and no interaction between the factors occurred (all $P>0.100$ ). Reaction time was shorter in the 300-ms SOA trials than in the 100-ms SOA trials ( $P$ $<0.001$ ), and shorter in the 500-ms SOA trials than in the $100-\mathrm{ms}$ SOA trials $(P<0.001)$ and $300-\mathrm{ms}$ SOA trials $(P$ $=0.004)$. Reaction time was shorter in the valid cue trials than in the bilateral cue trials $(P<0.001)$ and was shorter in the bilateral cue trials than in the invalid cue trials $(P<$ 0.001 ; Figure 2).

A planned ANOVA of the attentional effect, corresponding to the difference between reaction time in the valid cue trials and reaction time in the invalid cue trials, using the SOA and side indicated by the cue as factors, showed no main effect or interaction between the factors (all $P>0.100$ ).

To evaluate the importance of the differences observed between the experimental conditions we calculated the effect sizes. The effect sizes (square root of the F value divided by the square root of the number of participants; see Ref. 31, page 340) for the SOA, side indicated by the cue and their interaction were, $0.24,0.11$, and 0.10 , respectively (values of the order of 0.10 indicate a small difference, values of the order of 0.25 , a medium difference, and values of the order 
of 0.40 , a large difference; see Ref. 31, page 9). The size of the difference between the attentional effect produced by the cue indicating the right hemifield and the attentional effect produced by the cue indicating the left hemifield (difference between the means of two samples divided by their pooled standard deviation; see Ref. 32, page 3) was evaluated for each SOA. None of the values obtained was significant at the 0.05 level (that is, they fell inside the $95 \%$ confidence interval; see Ref. 32, page 8).

The present results do not indicate any favoring of the right (or left) side by voluntary attention.

\section{Experiment 2}

The rightward-orienting attentional mechanism of the right hemisphere is supposed to be more strongly mobilized in tasks requiring a higher level of attention. In this case, the hypothesized right side favoring by voluntary attention, which could not be demonstrated in the previous experiment, might appear by increasing the difficulty of the task.

In this experiment, the volunteers were tested in a more difficult task than the one of the previous experiment. They had to identify the shape of the target stimulus in addition
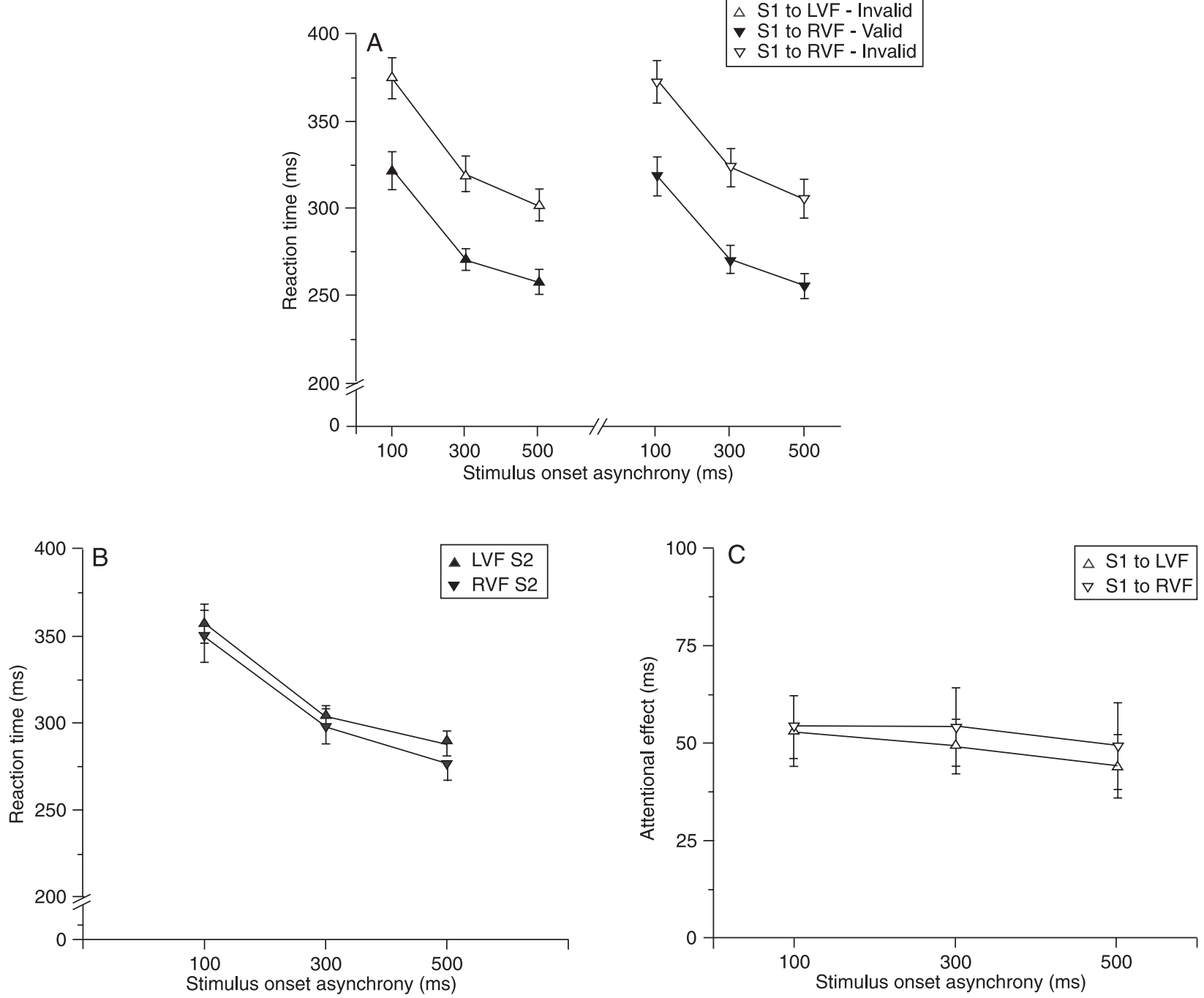

Figure 2. Reaction times at each stimulus onset asynchrony in Experiment 1. In the left side of Panel A, the central cue (S1) indicated the left hemifield and the target stimulus (S2) appeared in this hemifield (valid condition) or the opposite one (invalid condition). In the right side of Panel A, the S1 indicated the right hemifield and the S2 appeared in this hemifield or the opposite one. The post hoc Newman-Keuls test indicated that reaction time was globally longer in the invalid condition than in the valid condition $(P<0.001)$. In Panel $\mathrm{B}$, the S1 was bilateral and the S2 appeared in the left or right hemifield. The attentional effects (reaction time for the invalid condition minus reaction time for the valid condition) produced by S1 indicating the left side and by S1 indicating the right side, for each stimulus onset asynchrony, are shown in Panel C. Data are reported as means \pm SEM. LVF = left visual field. RVF $=$ right visual field. 
to its side (left or right) of appearance, and, depending on this shape, respond using the hand on the same side. As in the previous experiment, the location of the impending target was correctly or incorrectly indicated by a central cue in five-sixths of the trials. In the remaining trials, the central cue indicated that the target could appear on either side.

Again, a favoring of the right side by voluntary attention should be revealed by a shorter reaction time to the target on the right side than on the left side for the valid trials and/or a longer reaction time to the target on the left side than on the right side for the invalid trials. It might also be manifested by a shorter reaction time to the target on the right side than on the left side for the bilateral cue trials. An earlier appearance of the effects of right side-oriented attention might occur.

\section{Methods}

Participants. Twelve naive individuals with the characteristics described in Experiment 1 voluntarily participated in the experiment.

Procedure. The procedure was similar to that described in Experiment 1. Two target stimuli were now used. One was the vertical line previously used and the other was a small ring (0.3 degrees in diameter and a 0.004-degree wide margin), both lasting $34 \mathrm{~ms}$. A response should be given to the first stimulus (positive target), with the hand on the corresponding side, and no response should be given to the second stimulus (negative target). The positive target occurred in $66.67 \%$ of the trials $(33.3 \%$ of valid cue trials, $16.67 \%$ of invalid cue trials and $16.67 \%$ of bilateral cue trials) and the negative target occurred in $33.33 \%$ of the trials (all valid cue trials), with equal probability in the two hemifields. A lower percentage of negative target than positive target was used to avoid increasing the duration of the testing session, which would eventually make it tiresome for some volunteers.

In the second session, each of the "valid cue" conditions occurred a total of 48 times (24 times for the positive target and 24 times for the negative target), each of the "invalid cue" conditions occurred a total of 12 times (all of them for the positive target), and each of the "bilateral cue" conditions occurred a total of 12 times (all of them for the positive target).

Data analysis. The data were evaluated and analyzed as described in Experiment 1.

\section{Results}

The percentages of anticipation, inversion, false alarm, false alarm with inversion, and omission errors were 0.6, $0.1,2.0,<0.1$, and 1.6 , respectively.

A main effect of SOA [F2,22 = 123.06, $P<0.001]$ and of type of cue [F2,22 $=23.86, P<0.001]$, and an interaction between these two factors [F4,44 = 4.47, $\mathrm{P}=0.004$ ] occurred. A marginally significant interaction between SOA and target hemifield [F2,22 $=3.07, \mathrm{P}=0.067]$ and between
SOA, type of cue and target hemifield $[\mathrm{F} 4,44=2.20, \mathrm{P}=$ 0.085] also occurred. No main effect of target hemifield and no other interaction between factors occurred (all P > 0.1 ). Reaction time was shorter in the $300-\mathrm{ms}$ SOA trials than in the $100-m s$ SOA trials $(P<0.001)$, and shorter in the 500-ms SOA trials than in the 100-ms SOA trials $(P<$ $0.001)$ and $300-m s$ SOA trials $(P=0.010)$. Reaction time in the valid cue trials was not different from that in the bilateral cue trials $(P=0.180)$. Reaction times in both the valid and the bilateral cue trials were shorter than that in the invalid cue trials $(P<0.001$; Figure 3$)$.

A planned ANOVA of the attentional effect showed an interaction between the SOA and the side indicated by the cue $(P=0.037)$. The attentional effect produced by the cue indicating the left side did not differ between the three SOA (all $P>0.100$ ). The attentional effect produced by the cue indicating the right side did not differ between the 100-and the 300-ms SOAs ( $P>0.100)$; however, it was greater for the 500-ms SOA than for the 100-ms SOA $(P=0.017)$ and marginally greater for the 500-ms SOA than for the 300-ms $\operatorname{SOA}(P=0.060)$. The attentional effect produced by the cue indicating the right side did not differ from that produced by the cue indicating the left side for the 100- and the 300-ms SOAs; however, it was greater for the 500-ms SOA ( $P=$ 0.015; Figure 3).

The effect sizes for the SOA, side indicated by the cue and their interaction were $0.49,0.28$, and 0.57 , respectively. The size of the difference between the attentional effect produced by the cue indicating the right hemifield and the attentional effect produced by the cue indicating the left hemifield was evaluated for each SOA. Only the value obtained for the 500-ms SOA was significant at the 0.05 level.

The present results demonstrate a favoring of the right side by voluntary attention.

\section{Experiment 3a}

In this experiment, the volunteers were required to identify the shape of the target stimulus, to differentiate it from a distractor stimulus, in addition to identifying the side it occurred on. The presence of the distractor should increase the difficulty of the task compared to those used in the previous experiments, and, thus, the probability of occurrence of a mobilization of the rightward-orienting attentional mechanism of the right hemisphere strong enough to cause a lateral difference in performance. In addition, both the inhibitory action of voluntary attention in the valid trials and the facilitatory action of voluntary attention in the invalid trials should now present behavioral significance, as they would respectively reduce and increase the interference caused by the distractor on target processing. As these two actions would be laterally asymmetric, the attentional effect, which reflects their combined influence, should exhibit a greater lateral difference. The favoring of the right side 
by voluntary attention should manifest more clearly than in Experiment 2. As in the previous experiments, attention was oriented to the left, the right or to both visual hemifields by means of the central cue.

The favoring of the right side by voluntary attention would be indicated by a shorter reaction time to the target on the right side than on the left side for the valid and bilateral trials and a longer reaction time to the target on the left side than on the right side for the invalid trials.
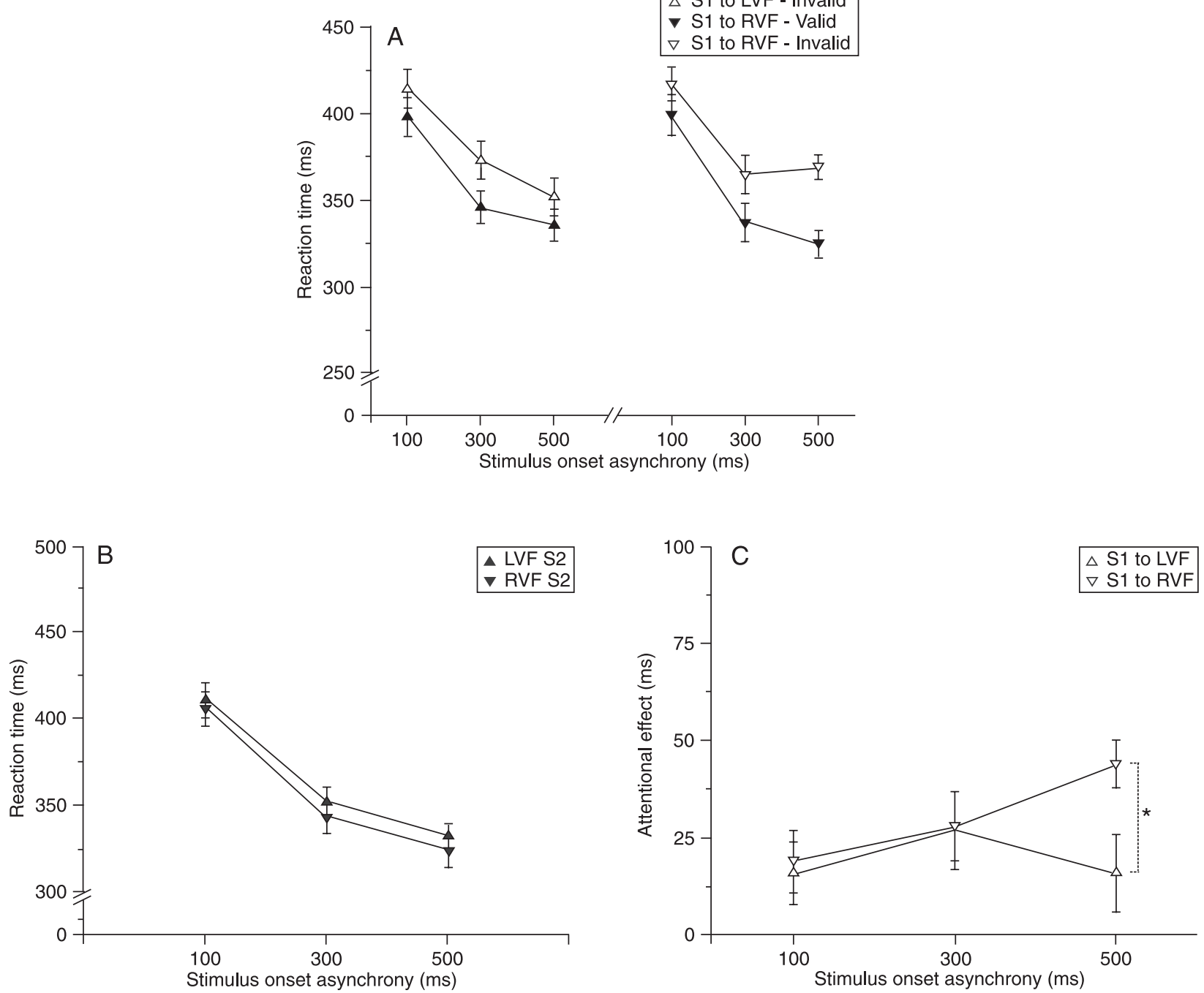

Figure 3. Reaction times at each stimulus onset asynchrony in Experiment 2. In the left side of Panel A, the central cue (S1) indicated the left hemifield and the target stimulus (S2) appeared in this hemifield (valid condition) or the opposite one (invalid condition). In the right side of Panel A, the S1 indicated the right hemifield and the S2 appeared in this hemifield or the opposite one. The post hoc Newman-Keuls test indicated that reaction time was globally longer in the invalid condition than in the valid condition $(P<0.001)$. In Panel B, the S1 was bilateral and the S2 appeared in the left or right hemifield. The attentional effects (reaction time for the invalid condition minus reaction time for the valid condition) produced by S1 indicating the left side and by S1 indicating the right side, for each stimulus onset asynchrony, are shown in Panel C. The post hoc Newman-Keuls test indicated that the attentional effect was greater when the cue pointed to the right side than when it pointed to the left side for the 500-ms stimulus onset asynchrony ( $\left.{ }^{*} P<0.015\right)$. Data are reported as means \pm SEM. LVF = left visual field; RVF = right visual field. 
0.04-degree wide margin). The other difference was that together with the target a distractor stimulus appeared on the opposite side, represented by the black horizontal line when the vertical line was used as target and by the black vertical line when the horizontal line was used as target.

Data analysis. The data were evaluated and analyzed as described in Experiment 1.

\section{Results}

The percentages of anticipation, inversion and omission errors were $0.8,3.9$, and 0.2 , respectively.

A main effect of SOA [F2,22 = 92.98, P < 0.001], type of cue $[F 2,22=47.34, P<0.001]$ and target hemifield $[F 1,11$ $=14.08, P=0.003$ ] occurred. An interaction between SOA and type of cue $[\mathrm{F} 4,44=7.47, \mathrm{P}<0.001]$ also occurred. Reaction time was shorter for the right hemifield target than for the left hemifield target. Reaction time was shorter in the 300-ms SOA trials than in the 100-ms SOA trials ( $P$ $<0.001$ ), and shorter in the 500-ms SOA trials than in the $100-\mathrm{ms}$ SOA trials $(P<0.001)$ and $300-\mathrm{ms}$ SOA trials $(P=$ $0.005)$. Reaction time was shorter in the valid cue trials than in the bilateral cue trials $(P<0.001)$ and was shorter in the bilateral cue trials than in the invalid cue trials $(P=0.009)$. For the 100-ms SOA, reaction time in the valid cue trials was shorter than that in the bilateral cue trials $(P<0.001)$; for the $300-$ and $500-\mathrm{ms}$ SOA, reaction time in the valid cue trials was shorter than that in the bilateral cue trials $(P<0.001$ and $P=0.007$, respectively) and reaction time in these trials was shorter than that in the invalid cue trials $(P=0.006$ and $P<0.001$, respectively; Figure 4).

A planned ANOVA of the attentional effect, using the SOA and the side indicated as factors, showed a main effect of SOA $[F 2,22=8.14, P=0.002]$ and side indicated $[F 1,11=$ 9.21, $P=0.011]$. The attentional effect was greater for the 300-ms SOA than for the 100- and 500-ms SOA ( $P=0.016$ and $P=0.002$, respectively). The attentional effect was greater when attention was oriented to the right side than when it was oriented to the left side (Figure 4).

The effect sizes for the SOA, side indicated by the cue and their interaction were $0.82,0.88$, and 0.41 , respectively. The size of the difference between the attentional effect produced by the cue indicating the right hemifield and the attentional effect produced by the cue indicating the left hemifield was evaluated for each SOA. Only the value obtained for the 500-ms SOA was significant at the 0.05 level.

The present results demonstrate a favoring of the right side by voluntary attention.

\section{Experiment 3b}

The greater effect of the right side indicating cue than of the left side indicating cue in Experiment 3a was attributed to a lateral asymmetry of voluntary attention. It could be questioned whether the result is not simply reflecting a sensory and/or motor lateral asymmetry, with right side stimulus/response processing being more efficient than left stimulus/response processing. To exclude this possibility, this experiment was replicated using only the bilateral central cue and the volunteers were informed that it was not relevant for task performance.

In this situation, we did not expect any lateral asymmetry in performance to occur. Reaction times should not differ between the right and the left sides for any SOA. The observation of a right side favoring would indicate that the result of Experiment 3a would have been caused, at least partially, by a lateral sensory and/or motor processing asymmetry.

\section{Methods}

Participants. Twelve naive individuals with the characteristics described in Experiment 1 voluntarily participated in the experiment.

Procedure. The procedure was similar to that described in Experiment 3a. The only difference was that the valid and invalid cue trials were replaced by bilateral cue trials and the volunteers were informed that the arrowheads that appeared centrally before the target were not relevant for task performance.

Data analysis. The data were evaluated as described in Experiment 1. Reaction time data were submitted to repeated measures ANOVA, with the SOA (100, 300, or $500 \mathrm{~ms})$ and hemifield of appearance of the target (left or right) as factors. When appropriate, these data were further analyzed by the Newman-Keuls test.

\section{Results}

The percentages of anticipation, inversion, and omission errors were $0.9,2.9$, and 0.1 , respectively.

A main effect of SOA [F2,22 $=41.74, \mathrm{P}<0.001]$ occurred. A marginally significant interaction between SOA and target hemifield $[F 2,22=2.64, P=0.093]$ also occurred. No main effect of target hemifield occurred. Reaction time was shorter in the 300-and the 500-ms SOA trials than in the 100-ms SOA trials $(P<0.001$ in both cases; Figure 5). In the 100-ms SOA trials, reaction time to the right hemifield target was longer than reaction time to the left hemifield target $(P=0.004)$.

The present results do not suggest the existence of any sensory or motor lateral asymmetry in the task requiring the identification of the shape of the target stimulus, to differentiate it from a distractor stimulus, and the identification of its side of occurrence.

\section{Experiment 4a}

In this experiment, the volunteers were required to identify the shape of the go target stimulus, to differentiate it from a distractor stimulus and from a no-go target stimulus, in addition to identifying its side of occurrence. The presence of the distractor should increase the difficulty of the task compared to those used in the previous experiments, increasing the mobilization of the right hemisphere 
rightward orienting attentional mechanism. In addition, both the inhibitory action of voluntary attention in the valid trials and the facilitatory action of voluntary attention in the invalid trials would now have behavioral significance, as they would respectively reduce and increase the interference with target processing caused by the distractor. The lateral difference of the capacity of orienting voluntary attention should express itself more strongly than in Experiment 2. As in the previous experiments, attention was oriented to the left, the right or to both visual hemifields by means of the central cue.

The favoring of the right side by voluntary attention would be indicated by a shorter reaction time to the target on the right side than on the left side for the valid and bilateral trials and a longer reaction time to the target on the left side than on the right side for the invalid trials.

\section{Methods}

Participants. Twelve naive individuals with the characteristics described in Experiment 1 voluntarily participated
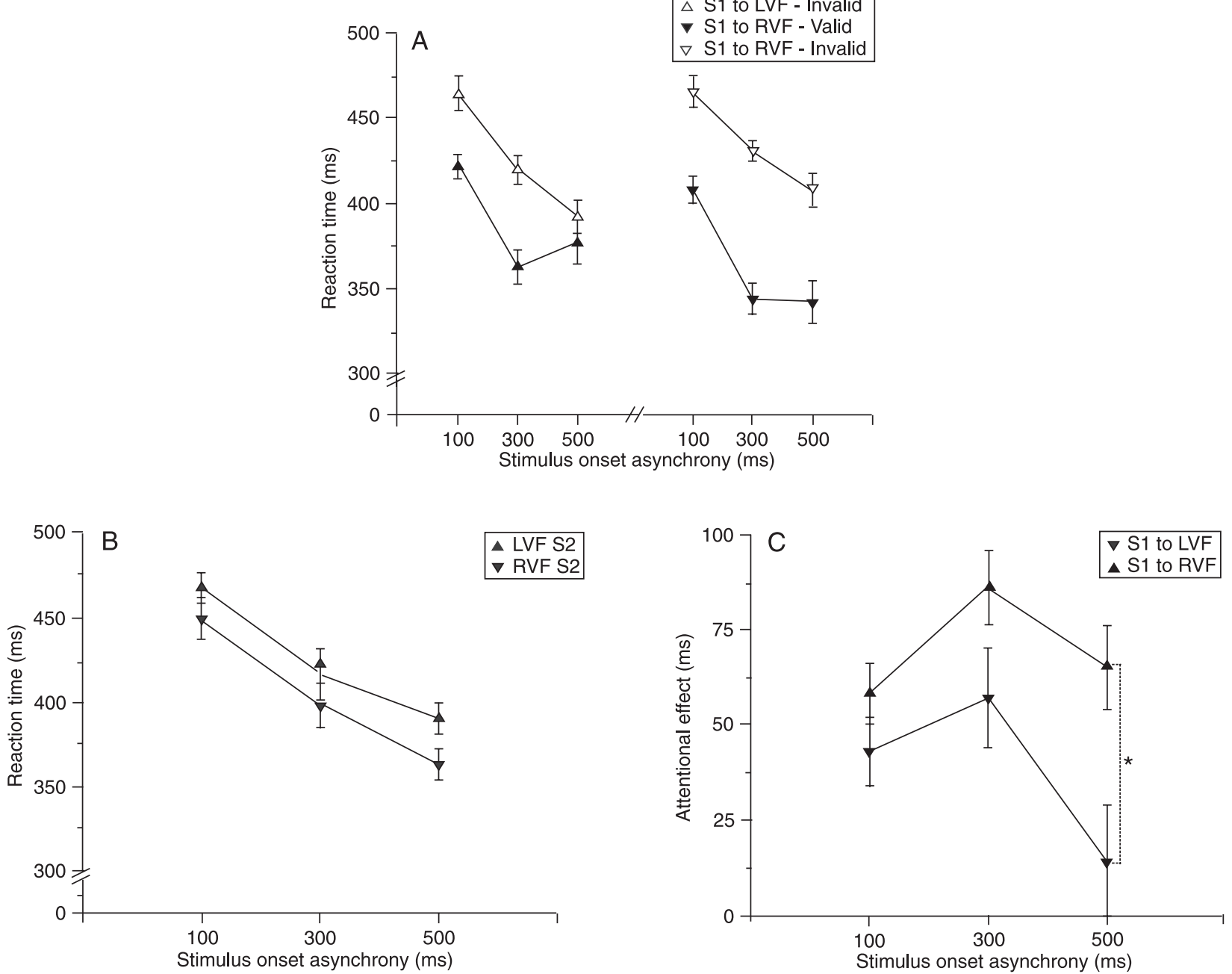

Figure 4. Reaction times at each stimulus onset asynchrony in Experiment 3a. In the left side of Panel A, the central cue (S1) indicated the left hemifield and the target stimulus (S2) appeared in this hemifield (valid condition) or in the opposite one (invalid condition). In the right side of Panel A, the S1 indicated the right hemifield and the S2 appeared in this hemifield or the opposite one. The post hoc Newman-Keuls test indicated that reaction time was globally longer in the invalid condition than in the valid condition $(P<0.001)$. In Panel B, the S1 was bilateral and the S2 appeared in the left or right hemifield. The attentional effects (reaction time for the invalid condition minus reaction time for the valid condition) produced by S1 indicating the left side and by S1 indicating the right side, for each stimulus onset asynchrony, are shown in Panel C. ANOVA indicated that the attentional effect was globally greater when the cue pointed to the right side than when it pointed to the left side $(P<0.011)$. The post hoc Newman-Keuls test indicated that the attentional effect was greater when the cue pointed to the right side than when it pointed to the left side particularly for the 500-ms stimulus onset asynchrony ( $\left.{ }^{*} P<0.006\right)$. Data are reported as means $\pm S E M$. LVF $=$ left visual field; RVF = right visual field. 
in the experiment.

Procedure. The procedure was very similar to that described in Experiment 2. There were only two differences: one was that for half of the volunteers the go target stimulus was the black vertical line and for the other half of the volunteers it was the black horizontal line ( 0.45 degrees in length and a 0.04-degree wide margin); the other difference was that, together with the go or no-go target, the distractor stimulus, represented by the black horizontal line when the vertical line was used as target and by the black vertical line when the horizontal line was used as target, appeared on the opposite side.

Data analysis. The data were evaluated and analyzed as described in Experiment 1.

\section{Results}

The percentages of anticipation, inversion, false alarm, false alarm with inversion, and omission errors were 1.1, $0.4,0.2,1.1$, and 0.7 , respectively.

A main effect of SOA [F2,22 = 155.17, P < 0.001], type of cue $[F 2,22=19.71, P<0.001]$ and target hemifield $[F 1,11=9.66, P=0.010]$, and an interaction between the first two factors $[F 4,44=2.92, P=0.032]$ occurred. No other interaction between factors (all P > 0.100) occurred. Reaction time was shorter for the right hemifield target than for the left hemifield target. Reaction time was shorter in the 300-ms SOA trials than in the 100-ms SOA trials ( $P<$ 0.001 ), and shorter in the 500-ms SOA trials than in the $100-\mathrm{ms}$ SOA trials $(P<0.001)$ and $300-\mathrm{ms}$ SOA trials $(P$ $=0.026)$. Reaction time in the valid cue trials tended to be

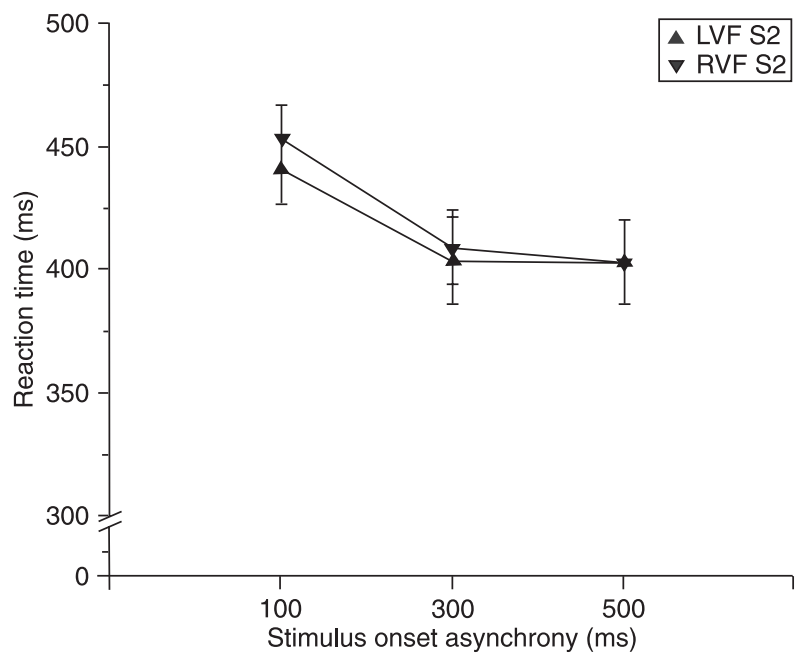

Figure 5. Reaction times at each stimulus onset asynchrony in Experiment $3 \mathrm{~b}$. The central double arrowhead preceded the target stimulus in all trials. The volunteers were told that it was not relevant for task performance. The target stimulus appeared randomly in the left or right hemifield. Data are reported as means \pm SEM. LVF = left visual field; RVF = right visual field. shorter than reaction time in the bilateral cue trials $(P=$ $0.083)$; reaction time in the bilateral cue trials was shorter than that in the invalid cue trials $(P<0.001)$. For the 100 and 300-ms SOA, reaction time in the valid cue trials was shorter than that in the bilateral cue trials (respectively, $\mathrm{P}<$ 0.001 and $P=0.022$ ) and reaction time in these trials was shorter than that in the invalid cue trials (respectively, $\mathrm{P}=$ 0.005 and $P<0.001$ ); for the $500-m s$ SOA, reaction time in the bilateral cue trials was shorter than that in the invalid cue trials $(P<0.001$; Figure 6$)$.

A planned ANOVA of the attentional effect showed a main effect of the side indicated by the cue $[F 1,11=10.09$, $P=0.009]$. The attentional effect produced by the cue indicating the right side was greater than that produced by the cue indicating the left side. The absence of any interaction between SOA and indicated side suggests that this lateral difference was similar for the three SOA (Figure 6).

The effect sizes for the SOA, side indicated by the cue and their interaction were $0.14,0.92$, and 0.35 , respectively. The size of the difference between the attentional effect produced by the cue indicating the right hemifield and the attentional effect produced by the cue indicating the left hemifield was evaluated for each SOA. The values obtained for the 100- and 300-ms SOA were significant at the 0.05 level.

The present results demonstrate a favoring of the right side by voluntary attention.

\section{Experiment 4b}

As considered for the results of Experiment $3 a$, the shorter reaction times to the right side target stimulus than to the left side go target stimulus observed in Experiment 4 a could have resulted from a sensory and/or motor lateral asymmetry. Although the results obtained in Experiment $3 \mathrm{~b}$ did not suggest the existence of such asymmetries, we decided to further investigate this possibility using the conditions of Experiment 4a. As in Experiment 3b, only the bilateral central cue was presented and the volunteers were informed that it was not relevant for task performance.

We did not expect any lateral asymmetry in performance to occur. Reaction times should not differ between the right and the left sides for any SOA. The observation of a right side favoring would indicate that the result of Experiment 4a might have been caused, at least partially, by a lateral sensory and/or motor processing asymmetry.

\section{Methods}

Participants. Twelve naive individuals with the characteristics described in Experiment 1 voluntarily participated in the experiment.

Procedure. The procedure was similar to that described in Experiment 4a. The only difference was that the valid and invalid cue trials were replaced by bilateral cue trials and the volunteers were informed that the arrowheads that 
appeared centrally before the target were not relevant for task performance.

Data analysis. The data were evaluated and analyzed as described in Experiment 3b.

\section{Results}

The percentages of anticipation, inversion, false alarm, false alarm with inversion, and omission errors were 0.6, $0.6,0.2,0.6$, and 0.8 , respectively.

A main effect of SOA [F2,22 $=145.54, \mathrm{P}<0.001]$ occurred. No main effect of target hemifield or interaction between the two factors occurred.

Reaction time was shorter in the 300- and the 500-ms SOA trials than in the $100-\mathrm{ms}$ SOA trials $(\mathrm{P}<0.001$ in both
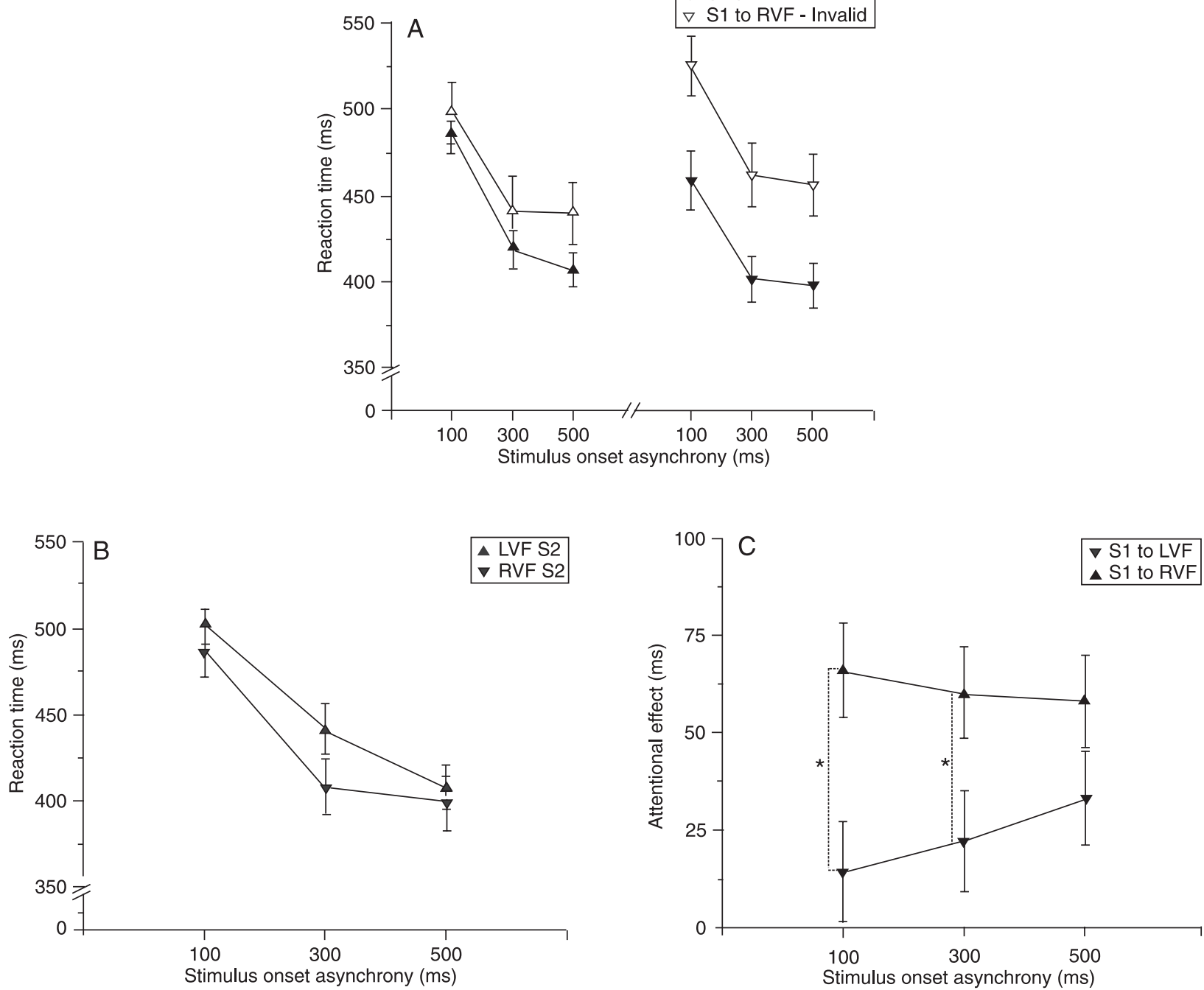

Figure 6. Reaction times at each stimulus onset asynchrony in Experiment 4a. In the left side of Panel A, the central cue (S1) indicated the left hemifield and the target stimulus (S2) appeared in this hemifield (valid condition) or the opposite one (invalid condition). In the right side of Panel A, the S1 indicated the right hemifield and the S2 appeared in this hemifield or the opposite one. The post hoc Newman-Keuls test indicated that reaction time was globally longer in the invalid condition than in the valid condition $(P<0.001)$. In Panel B, the S1 was bilateral and the S2 appeared in the left or right hemifield. The attentional effects (reaction time for the invalid condition minus reaction time for the valid condition) produced by S1 indicating the left side and by S1 indicating the right side, for each stimulus onset asynchrony, are shown in Panel C. ANOVA indicated that the attentional effect was globally greater when the cue pointed to the right side than when it pointed to the left side $(P<0.009)$. The post hoc Newman-Keuls test indicated that the attentional effect was greater when the cue pointed to the right side than when it pointed to the left side particularly for the 100-and the 300-ms stimulus onset asynchronies (respectively, $\mathrm{P}<0.002$ and $\mathrm{P}<0.013$ ). Data are reported as means $\pm \mathrm{SEM}$. LVF $=$ left visual field; RVF $=$ right visual field. 
cases; Figure 7).

The present results do not suggest the existence of any sensory or motor lateral asymmetry in the task requiring the identification of the shape of the go target stimulus, to differentiate it from a distractor stimulus and a no-go target stimulus, and the identification of its side of occurrence.

\section{Discussion}

This study investigated the possible existence of a favoring of the right side by voluntary attention. Evidence supporting such a hypothesis was obtained in three of the four experiments. A common factor in these three experiments was the relative difficulty of the tasks used to test the volunteers.

An important effect of voluntary attention was obtained in all four experiments of this study. On average, the attentional effect (difference between reaction time in the valid cue trials and reaction time in the invalid cue trials) was 51, 30, 54, and $42 \mathrm{~ms}$ in Experiments 1, 2, 3a, and $4 a$, respectively. In all cases, it was already present in the 100-ms stimulus onset asynchrony. In Experiments 1, 2, and $4 a$, it did not change much across the next two stimulus onset asynchronies, but in Experiment 3a, it increased and then returned to close to its initial value.

The effects produced by voluntary attention orienting to the right side did not differ from those produced by voluntary attention orienting to the left side in Experiment 1, in which the volunteers simply had to identify the location of occurrence of the target stimulus. In Experiment 2, in which the volunteers had to identify the location and the shape of the positive target, a greater effect was observed at the 500-ms stimulus onset asynchrony when voluntary attention was oriented to the right side.

The effect produced by voluntary attention orienting to the right side was greater than that produced by voluntary attention orienting to the left side at the 500-ms SOA in Experiment $3 \mathrm{a}$ and at the 100- and 300-ms SOA in Experiment $4 a$. In Experiment 3a, the volunteers had to differentiate the target from a distractor stimulus, which appeared simultaneously at the contralateral location, in addition to identifying its location, and in Experiment 4a, they had to differentiate the positive target from the distractor stimulus, which appeared simultaneously at the contralateral location, and the negative target in addition to identifying its location. Differently from Experiment 2, in Experiments $3 a$ and 4a, a tendency to the occurrence of a lateral difference could be observed for the remaining SOA.

The results of Experiment 2 for the 500-ms SOA, of Experiment $3 a$ for the same SOA and of Experiment $4 a$ for the 100 - and $300-m s$ SOA strongly suggest that voluntary attention orienting tends to favor the right side of space. This conclusion is further supported by the evidence that visuomotor processing is not laterally asymmetric in the tasks used. Thus, in Experiment 2 no lateral difference in performance occurred for the 100- and 300-ms SOA.

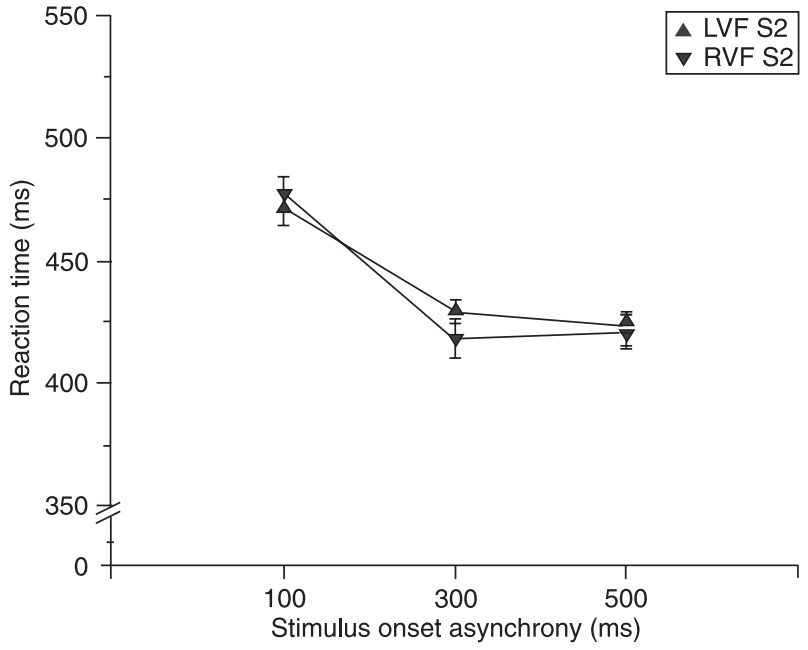

Figure 7. Reaction times at each stimulus onset asynchrony in Experiment $4 \mathrm{~b}$. The central double arrowhead preceded the target stimulus in all trials. The volunteers were told that it was not relevant for task performance. The target stimulus appeared randomly in the left or right hemifield. Data are reported as means \pm SEM. LVF = left visual field; RVF = right visual field.

Similarly, in Experiments $3 \mathrm{~b}$ and $4 \mathrm{~b}$, in which voluntary attention was not explicitly controlled, no lateral difference in performance appeared for any SOA. The importance of these controls is made clear when considering that the effects of voluntary attention orienting to the right side were obtained by subtracting valid right side reaction times from invalid left side reaction times and the effects of voluntary attention orienting to the left side were obtained by subtracting valid left side reaction times from invalid right side reaction times. Shorter right side reaction times than left side reaction times, due to a visuomotor processing favoring the right side, would lead to the same general results as those obtained in this study.

We expected a faster development of the effects produced by voluntary attention orienting to the right side of space than of the effects produced by voluntary attention orienting to the left side of space. No lateral difference was observed, however, in Experiments 1, 2, 3a, and 4a. Perhaps by using a greater degree of short SOAs such a difference could be demonstrated. This possibility should be tested in future experiments.

The double hemispheric control of orienting to the right side, as opposed to the single hemispheric control of orienting to the left side $(21-23,25)$, might be responsible for the right side attentional favoring. The smaller hemispheric asymmetry of voluntary attention mechanisms compared to automatic attention mechanisms might well explain the difference between the results obtained in Experiments 1 and 2 and the results obtained by Castro-Barros et al. (17) using the same tasks and target stimuli.

The somewhat different results obtained in the four 
experiments of this study might be related to the relative attentional demands of their respective tasks. The task of simply identifying the location of the target stimulus, used in Experiment 1, might have been too easy to significantly mobilize the right hemisphere mechanism, which controls voluntary attention orienting to the right side. In the case of Experiment 2, the task of identifying the location and the shape of the target stimulus might have been difficult enough to significantly mobilize this right hemisphere mechanism. In Experiments $3 a$ and $4 a$, the presence of the contralateral distractor further increased, presumably, the demand for attentional resources and guaranteed more involvement of this right hemisphere mechanism.

The present results agree with those of Rhodes and Robertson (27). The shorter reaction time observed by these authors when voluntary attention was oriented to the right side compared to when it was oriented to the left side, in the valid condition, could, however, simply reflect the linguistic nature of their targets (see, for example, Ref. 29). However, this kind of explanation is obviously not a consideration in the case of the results reported here since geometric figures were used as targets.

At this point, one can only speculate concerning the reason for the opposite results obtained by Evert et al. (28). As described before, in the second experiment of these authors in which the target was associated with a contralateral distractor, the invalid cue produced a cost when pointing to the left visual hemifield but not when pointing to the right hemifield. It is possible that since these authors used letters as stimuli, their volunteers developed a left-right scanning tendency when these letters were presented bilaterally. This tendency might have compensated the contralateral inhibitory influence of voluntary attention orienting to the right side, abolishing its behavioral cost.

Another potential explanation for the difference between the results of Evert et al. (28) and those of Rhodes and Robertson (27) and the present results is that the observed right side favoring was related to the specific spatial frequencies characterizing the relevant features of the targets. A fairly large body of evidence exists suggesting that the right hemisphere is dominant for processing relatively low spatial frequencies and the left hemisphere for processing relatively high spatial frequencies (see Ref. 33). The absence of any lateral asymmetry in part of the experiments in which the same target stimuli were used argues against this hypothesis. In addition, it should be noted that the manifestation of the lateral asymmetries related to the relative spatial frequencies of the target stimuli depends on discrimination of this type of feature as a requirement for task performance (34). There is no reason to suppose this was the case in the studies under consideration.

The smaller attentional effect for the target location and shape identification task in Experiment 2 than for the location identification task in Experiment 1 replicates the findings of Castro-Barros et al. (17). These investigators considered two possible explanations for this result. One was that the difference could be related to a less efficient use of the cue to orient attention, due to the need to also store in working memory critical information regarding the significance of the two target stimuli. The other was that the difference could reflect a higher criterion for responding to the target in the former task, particularly in the valid condition, so as to avoid responding incorrectly to the no-go target. The relatively strong attentional effect observed in Experiment $3 a$, in which the participants had to store in working memory which of the two simultaneously presented stimuli was the target appears to support the latter interpretation.

The present study presented robust evidence that indicates that voluntary visual attention tends to favor the right side, in accordance with the large neural network (left and right hemisphere networks) available to control its orientation to the right side as opposed to the smaller neural network (right hemisphere circuit) available to control its orientation to the left side.

\section{Acknowledgments}

Research supported by CNPq, CAPES and FAPESP.

\section{References}

1. Shulman GL, Astaviev SV, Corbetta M. Two cortical systems for the selection of visual stimuli. In: Posner MI (Editor), Cognitive neuroscience of attention. New York: The Gilford Press; 2004. p 114-126.

2. Brefczynski JA, DeYoe EA. A physiological correlate of the 'spotlight' of visual attention. Nat Neurosci 1999; 2: 370374.

3. Hillyard SA, Anllo-Vento L. Event-related brain potentials in the study of visual selective attention. Proc Natl Acad Sci U S A 1998; 95: 781-787.

4. Carrasco M, Williams PE, Yeshurun Y. Covert attention increases spatial resolution with or without masks: support for signal enhancement. J Vis 2002; 2: 467-479.
5. Dosher BA, Lu ZL. Noise exclusion in spatial attention. Psychol Sci 2000; 11: 139-146.

6. Kastner S. Attentional response modulation in the human visual system. In: Posner MI (Editor), Cognitive neuroscience of attention. New York: The Gilford Press; 2004. p 144-156.

7. Kastner S, De Weerd P, Desimone R, Ungerleider LG. Mechanisms of directed attention in the human extrastriate cortex as revealed by functional MRI. Science 1998; 282 : 108-111.

8. Lachter J, Forster KI, Ruthruff E. Forty-five years after Broadbent (1958): still no identification without attention. Psychol Rev 2004; 111: 880-913. 
9. Ling S, Carrasco M. When sustained attention impairs perception. Nat Neurosci 2006; 9: 1243-1245.

10. Reynolds JH, Chelazzi L, Desimone R. Competitive mechanisms subserve attention in macaque areas V2 and V4. $J$ Neurosci 1999; 19: 1736-1753.

11. Serences JT, Yantis S, Culberson A, Awh E. Preparatory activity in visual cortex indexes distractor suppression during covert spatial orienting. J Neurophysiol 2004; 92: 35383545.

12. Silver MA, Ress D, Heeger DJ. Neural correlates of sustained spatial attention in human early visual cortex. $J$ Neurophysiol 2007; 97: 229-237.

13. Somers DC, Dale AM, Seiffert AE, Tootell RB. Functional MRI reveals spatially specific attentional modulation in human primary visual cortex. Proc Natl Acad Sci U S A 1999; 96: 1663-1668.

14. Tootell RB, Hadjikhani N, Hall EK, Marrett S, Vanduffel W, Vaughan JT, et al. The retinotopy of visual spatial attention. Neuron 1998; 21: 1409-1422.

15. Treue $\mathrm{S}$. Neural correlates of attention in primate visual cortex. Trends Neurosci 2001; 24: 295-300.

16. Corbetta M, Shulman GL. Control of goal-directed and stimulus-driven attention in the brain. Nat Rev Neurosci 2002; 3: 201-215.

17. Castro-Barros BA, Righi LL, Grechi G, Ribeiro-do-Valle LE. Interlateral asymmetry in the time course of the effect of a peripheral prime stimulus. Brain Cogn 2008; 66: 265-279.

18. Pollmann S. A pop-out induced extinction-like phenomenon in neurologically intact subjects. Neuropsychologia 1996; 34: 413-425

19. Pollmann S. Extinction-like effects in normals: independence of localization and response selection. Brain Cogn 2000; 44: 324-341.

20. Steinman BA, Steinman SB, Lehmkuhle S. Visual attention mechanisms show a center-surround organization. Vision Res 1995; 35: 1859-1869.

21. Corbetta M, Miezin FM, Shulman GL, Petersen SE. A PET study of visuospatial attention. J Neurosci 1993; 13: 12021226.

22. Kim YH, Gitelman DR, Nobre AC, Parrish TB, LaBar KS,
Mesulam MM. The large-scale neural network for spatial attention displays multifunctional overlap but differential asymmetry. Neuroimage 1999; 9: 269-277.

23. Nobre AC, Sebestyen GN, Gitelman DR, Mesulam MM, Frackowiak RS, Frith $C D$. Functional localization of the system for visuospatial attention using positron emission tomography. Brain 1997; 120 (Part 3): 515-533.

24. Mesulam MM. Spatial attention and neglect: parietal, frontal and cingulate contributions to the mental representation and attentional targeting of salient extrapersonal events. Philos Trans R Soc Lond B Biol Sci 1999; 354: 1325-1346.

25. Grosbras MH, Paus T. Transcranial magnetic stimulation of the human frontal eye field: effects on visual perception and attention. J Cogn Neurosci 2002; 14: 1109-1120.

26. Barthelemy S, Boulinguez P. Orienting visuospatial attention generates manual reaction time asymmetries in target detection and pointing. Behav Brain Res 2002; 133: 109-116.

27. Rhodes DL, Robertson LC. Visual field asymmetries and allocation of attention in visual scenes. Brain Cogn 2002; 50: 95-115.

28. Evert DL, Glinchey-Berroth R, Verfaellie M, Milberg WP. Hemispheric asymmetries for selective attention apparent only with increased task demands in healthy participants. Brain Cogn 2003; 53: 34-41.

29. Mondor TA, Bryden MP. On the relation between visual spatial attention and visual field asymmetries. Q J Exp Psychol A 1992; 44: 529-555.

30. Oldfield RC. The assessment and analysis of handedness: the Edinburgh Inventory. Neuropsychologia 1971; 9: 97113.

31. Aron A, Aron EN. Statistics for psychology. Upper Saddle River: Prentice-Hall; 1999.

32. Coe R. It's the effect size, stupid. What effect size is and why it is important. British Educational Research Association Annual Conference. Exeter, 12-14 September. 2002.

33. Ivry RB, Robertson LC. The two sides of perception. Cambridge: The MIT Press; 1998.

34. Proverbio AM, Zani A, Avella C. Hemispheric asymmetries for spatial frequency discrimination in a selective attention task. Brain Cogn 1997; 34: 311-320. 